\title{
Early catheter removal after transurethral resection of the prostate
}

\author{
Neville D Perera' and A C N Nandasena ${ }^{2}$ \\ (Index words: Short irrigation period, low complication rate, prostates weighing less than $25 \mathrm{~g}$ )
}

\begin{abstract}
Introduction Post-operative care of transurethral resection of the prostate (TURP) includes prolonged bladder irrigation that places a heavy burden on the nursing staff and a substantial strain on the budget. There is a trend towards early catheter removal after TURP even to the extent of performing it as a day case. We explored the feasibility and limitations of early catheter removal after TURP in our unit.
\end{abstract}

Design Prospective study.

Setting Department of Urology, The National Hospital of Sri Lanka (NHSL), Colombo.

Patients and Methods The study was in a tertiary referral centre (NHSL), on 65 patients with a mean age of 67.5 years who underwent TURP for mild to moderate enlargement of the prostate, less than $25 \mathrm{~g}$, with lower urinary tract symptoms. Post-operative irrigation was maintained by diuretics at operation or a short term saline irrigation in the operating theatre.

Results 17 patients developed clot retention in the ward that was managed by irrigation for 12 to $24 \mathrm{~h} .62$ patients who had clear or minimally blood-stained urine were tried without catheter after $24 \mathrm{~h}$. Only two failed to pass urine. Patients without other complications were discharged from hospital after 1 or 2 successful voidings on the same day. There were no readmissions with complications.

Conclusions This study supports the feasibility of early catheter removal after a short irrigation period in TURP in the majority of patients with mild to moderate enlargement of the prostate without significantly increasing postoperative complications.

\section{Introduction}

Transurethral resection of the prostate (TURP) is still considered as the gold standard of surgical treatment of bladder outflow obstruction due to prostatic enlargement (1). Post-operative bladder irrigation is traditionally used for 12 to $24 \mathrm{~h}$ and catheter removal with a voiding trial is recommended after 2 to 5 days. This imposes a heavy burden on the nursing staff and a substantial financial burden (2), between $\$ 3545$ and 5930 in the USA (3) and estimated at $\$ 600$ to 800 in the Sri Lankan private sector. About $30 \%$ to $35 \%$ of this is spent on hospital accommodation and 5 to $7 \%$ on post-operative irrigation. In the developed world during the past decade there have been several trials of early catheter removal with shortening of the post-operative irrigation period and the hospital stay (1-6). This study was planned to assess the possibility of applying this method to patients undergoing TURP for mild to moderate enlargement of prostate gland, with a view to identifying the practical problems in a developing country.

\section{Methods}

This prospective study was done in our unit in the NHSL where about 500 TURPs are performed annually. Patients with moderate to severe lower urinary tract symptoms and clinically and ultrasonically assessed mild to moderate enlargement of the prostate (less than $25 \mathrm{~g}$ resected weight) were included in the study. Patients were told about the new protocol and the management plan for post-operative complications such as clot retention.

Preoperative preparation was carried out at the outpatient clinic and admission took place $24 \mathrm{~h}$ before surgery. TURP was performed by the urologist, or senior registrars, under spinal anaesthesia. Standard trilobar resection with $22 \mathrm{~F}$ resectoscope was used with routine precautions taken to achieve maximum haemostasis without undue prolongation of resection time.

All patients were irrigated with saline in the recovery room. When the effluent was clear or light pink irrigation was stopped and $40 \mathrm{mg}$ of frusemide was administered eight-hourly with encouragement to drink 2 to 31 of fluids during next $24 \mathrm{~h}$. In case of heavy haematuria or clot retention irrigation was re-established for a further period of 6 to $12 \mathrm{~h}$, until the effluent became clear.

Thereafter a voiding trial without catheter (TWOC) was attempted. Patients with clear urine after 1 to 2 voiding episodes and achieving clinically confirmed bladder emptying were discharged on the same day. Those who failed were catheterised for a further $48 \mathrm{~h}$ before a second TWOC. Patients were asked at their next clinic visit regarding late complications such as haematuria, retention and readmission to other units.

\section{Results}

65 patients with a mean age of 67.5 years and resected prostatic weight less than $25 \mathrm{~g}$ were included in the study. 3 patients were excluded owing to perioperative complica-

'Urological Surgeon and ${ }^{2}$ Senior Urological Registrar, National Hospital of Sri Lanka, Colombo. (Received 10 December 2001, revised version accepted 12 January 2002. Corresponding author NDP, email: nevi603@sltnet.lk; telephones +941867061 and +941882655 ) 
tions such a capsular perforation and heavy venous bleeding after one hour, rendering them unsuitable for termination of irrigation and early catheter removal.

Of the 62 patients included, 45 maintained clear catheter drainage and successfully voided after the catheter was removed at $24 \mathrm{~h}$. The other 17 patients developed clot retention in the ward after stopping irrigation. However, re-establishment of irrigation for a further 6 to $12 \mathrm{~h}$ cleared the clots allowing catheter removal at $24 \mathrm{~h}$ as planned. 7 patients failed to void, a failure rate of $11.3 \%$ (7/62). Failures were catheterised and only two patients had retained clots. TWOC was repeated after 48 hours and only 2 out of 7 failed to pass urine. They were discharged with an indwelling catheter for a final trial in 3 to 4 weeks.

None of the patients developed the TURP syndrome or required blood transfusion in this series, but 8 patients needed prolonged hospital stay for urinary tract infection (5 patients), myocardial instability (2) and severe urge incontinence (1). On discharge the patients living far from NHSL were given instructions to inform the local surgeons immediately in case of heavy haematuria or retention. No re-admissions were recorded in later follow up.

\section{Discussion}

Standard textbook recommendations for irrigation and catheter removal are $24 \mathrm{~h}$ and 2 to 5 days after surgery (1). Early cessation of irrigation and catheter removal have been tested repeatedly without considering the prostatic volume as a selection criterion. We decided to consider smaller prostatic weight as an inclusion criterion in this preliminary study because of the significantly higher tendency for post-operative haemorrhage in larger prostates, lack of proper community urological support, and presence of a large number of moderately enlarged prostates in day-today practice.

Although the clot retention rate after the early stoppage of irrigation is rather high in this series $(27.4 \%)$, in 10 out of these 17 patients who developed catheter clot retention, irrigation for a further 6 to $8 \mathrm{~h}$ ended in successful TWOC within $24 \mathrm{~h}$. Of the 7 patients who failed the $24 \mathrm{~h}$ trial, only 2 had clots and 5 probably had local oedema of the prostatic bed or bladder hypotonia. All except 2 were successful in passing urine at the second TWOC in $48 \mathrm{~h}$, leaving only 2 true failed catheter trials both probably due to detrusor hypotonia.

Failures have to be considered against the comfort enjoyed by the majority of successful cases and the reduced work-load of the staff by early catheter removal. The final success rate of early catheter removal (55/62; $88.7 \%$ ) was either better than or similar to other studies $(4,6)$. The low complication and readmission rate for retention could also be related to the low resected prostate weight.

\section{Conclusions}

Our study confirms the safety of an irrigation-free and early catheter removal policy after TURP in prostates weighing $25 \mathrm{~g}$ or less, with a significant reduction of post-operative burden on the staff and budget. Our study encourages larger studies of this protocol.

\section{References}

1. Feldstein MS, Benson NA. Early catheter removal and reduced length of hospital stay following transurethral prostatectomy; a retrospective analysis of 100 consecutive patients. Journal of Urology 1988; 140: 1403-6.

2. Agrawal SK, Kumar ASV. Early removal of catheter following transurethral resection of the prostate. British Journal of Urology 1993; 72: 928-9.

3. Koh KBH, MacDermott JP, Smith PH, Whelan P. Early catheter removal following transurethral prostatectomy - impact on length of hospital stay. British Joumal of Urology 1994; 74: 61-3.

4. Dodds L, Lowson PS, Crothwait AH, Wells GR. Early catheter removal: a prospective study of 100 consecutive patients undergoing transurethral resection of the prostate. British Journal of Urology 1995; 75: 755-7.

5. Muellar M, Zeidman EJ, Desmond PM, Thompson IM, Optenberg SA, Wasson J. Reduction of length of stay and cost of transurethral resection of the prostate by early catheter removal. British Journal of Urology 1996; 78: 893-6.

6. Gordon NSI. Catheter free same-day surgery in transurethral resection of the prostate. Journal of Urology 1998; 160: 1709-12. 